\title{
Characteristics of PEGylated Polydiacetylene Liposome and its Inclusion Complex Formation with $\alpha$-Cyclodextrin
}

\author{
Hye Choi and Joon Sig Choi ${ }^{*}$ \\ Department of Biochemistry, College of Natural Sciences, Chungnam National University, Daejeon 305-764, Korea \\ "E-mail: joonsig@cnu.ac.kr \\ Received July 3, 2013, Accepted July 25, 2013
}

\begin{abstract}
Diacetylene lipid monomers possess the capability to self-assemble into vesicles via polymerization under ultraviolet irradiation, resulting in the formation of polydiacetylene (PDA) liposomes. Exposure of the polymerized vesicles to external stimuli is known to induce a unique blue-to-red color transition. The cyclic oligosaccharide $\alpha$-cyclodextrin known for its use in many applications, such as drug delivery, purification, and stimulus sensing, is able to form an inclusion complex with poly(ethylene glycol) (PEG) in aqueous solution. In this study, we prepared polymeric liposomes with PEG (PEG-PDA) with the aim of improving the stability of the vesicles and colorimetric response toward $\alpha$-cyclodextrin. We demonstrated that PEG-PDA liposome displays unique characteristics compared with native PDA liposome and it also shows apparent chromic properties of the inclusion complex formation with $\alpha$-cyclodextrin.
\end{abstract}

Key Words : Polydiacetylene, Liposome, Self-inclusion complex, $\alpha$-Cyclodextrin, Poly(ethylene glycol)

\section{Introduction}

Non-covalent bonds, which are crucial intermolecular forces involved in the assembly of supramolecular complexes, are generally based on host-guest interactions. These forces are involved in maintaining three-dimensional structures of macromolecules via hydrogen bonding, electrostatic forces, hydrophobic interactions, and van der Waals interactions. Owing to its structural properties, cyclodextrin continues to be one of the most studied host molecules, and has found application in various fields such as drug delivery ${ }^{1,2}$ and stimulus sensing. ${ }^{3}$ Cyclodextrins are cyclic oligosaccharides consisting of six, seven, or eight glucose units joined by $\alpha$ 1,4-linkages, termed $\alpha, \beta$, and $\gamma$-cyclodextrin, respectively. The molecules are identical in height but vary in diameter and volume based on unit number. ${ }^{4} \mathrm{X}$-ray analysis has demonstrated that the $\mathrm{C} 2$ and $\mathrm{C} 3$ ' hydroxyl groups are outward-facing, rendering the cyclodextrin ring exterior generally hydrophilic, whereas the $\mathrm{C} 3$ and $\mathrm{C} 5$ ' hydrogens and ether oxygen atoms situated on the interior provide a somewhat hydrophobic central cavity. ${ }^{5}$ The formation of inclusion complexes in which the cavity of a "host" compound is occupied by a second molecule "guest" between cyclodextrin and poly(ethylene glycol) (PEG) was first described in $1990 .^{6}$ A subsequent report by Goh et al. demonstrated that low-molecular weight PEG is able to form crystalline inclusion complexes with $\alpha$-and $\gamma$-cyclodextrin. ${ }^{7}$

We have previously reported the inclusion complex formation between PEG-conjugated polydiacetylene (PDA) micelles and $\alpha$-cyclodextrin ${ }^{8}$ in which the 1,4-addition reaction of diacetylene monomers via UV irradiation-initiated polymerization generates PDA, a self-assembling lipid with an alternating ene-yne polymeric structure. ${ }^{9,10}$ Because the polymerization does not require a chemical initiator or catalyst, this process has the added advantage of avoiding the need for a purification step. The conjugated groups of PDA undergo further conformational changes in response to external stimuli such as heat, ${ }^{11-13}$ organic solvents,,${ }^{14,15}$ mechanical stress, ${ }^{16-18}$ or biomolecule addition, ${ }^{19-24}$ resulting in distinctive chromic changes that can be exploited for the development of colorimetric chemosensors or biomolecular sensors. Herein, we constructed a PEGylated PDA liposomal nanovesicle and characterized its unique properties along with the inclusion complex formation by PEGinduced interaction with $\alpha$-cyclodextrin.

\section{Experimental Sections}

Materials and Reagents. 10,12-Pentacosadiynoic acid (PCDA), $N, N$-dimethylformamide, (DMF, anhydrous $99.8 \%$ ), and $N, N$-diisopropylethylamine (DIPEA) were purchased from Sigma-Aldrich (Korea). Methoxy(polyethylene glycol)amine (SUNBRIGHT MEPA-20H, MW 2,000) was obtained from NOF (Japan). $N$-hydroxybenzotriazole $(\mathrm{HOBt}$ ) and 2-(1H-benzotriazole-1-yl)-1,1,3,3-tetramethyluronium (HBTU) were purchased from Anaspec Inc.(San Jose, CA, USA). $\alpha-$ Cyclodextrin was purchased from Bio-Research Corporation (Yokohama, Japan). Ultrapure water $(18.3 \mathrm{M} \Omega / \mathrm{cm})$ from the Human Ultra Pure System (Human Corp., Korea) was used.

Preparation of mPEG-induced PCDA Liposomes (10 mol \% mPEG-PCDA Liposomes). Methoxy poly(ethylene glycol)-conjugated PCDA monomer (mPEG-PCDA) was successfully synthesized according to our previously reported method. ${ }^{8}$ The molecular weight of the mPEG-PCDA monomer lipid was measured as $2645.1 \mathrm{~g} / \mathrm{mol}$ by MALDITOF mass spectrometry. ${ }^{8}$ A mixture of mPEG-PCDA conjugate and PCDA (1:9 molar ratio) was prepared in chloroform, followed by solvent evaporation under a stream of $\mathrm{N}_{2}$ 
gas. Distilled water was added to yield a total PCDA lipid concentration of $1 \mathrm{mM}$ and the resulting suspension was then sonicated using a bath-type sonicator for $15 \mathrm{~min}$. A control solution of liposome comprised of PCDA only was prepared in the same manner as above, but with heating at 80 ${ }^{\circ} \mathrm{C}$ for $15 \mathrm{~min}$ prior to sonication. After sonication, the solutions were immediately filtered using a $0.8 \mu \mathrm{m}$ syringe filter and stored at $4{ }^{\circ} \mathrm{C}$ for $12 \mathrm{~h}$. Liposome polymerization was performed by irradiation in a UV chamber reactor (RMR-600, $254 \mathrm{~nm}$ ) for $90 \mathrm{~s}$ at room temperature. Wavelength scanning in the visible region was performed using a UV-vis spectrophotometer (Ultrospec 2100 pro UV/visible spectrophotometer, Amersham Pharmacia Biotech, Cambridge, UK).

Characterization of Polymeric Liposomes. The hydrodynamic diameters and zeta potential values were determined using a Zetasizer Nano ZS system (Malvern Instruments, UK). Sizes of the liposome particles were measured using Dispersion Technology Software 4.30, with data analysis performed in automatic mode. The refractive index and viscosity of ultrapure water was measured at $25{ }^{\circ} \mathrm{C}$, and determined to be 1.33 and $0.89 \mathrm{cP}$, respectively. The values are presented as the average size \pm standard deviation of three runs.

Interaction of PDA and $\alpha$-Cyclodextrin. Vesicles composed of mPEG-PCDA:PCDA (10:90, mol\%) were prepared in distilled water and subsequently polymerized by UV irradiation at $254 \mathrm{~nm}$ for 90s. $\alpha$-Cyclodextrin was then added to the polymerized liposome solution at a final concentration of $0.5 \mathrm{mM}$. Various concentration-dependent color changes were observed by using UV-vis spectroscopy.

Differential Scanning Calorimetry (DSC). DSC experiments were carried out using a DSC 1 instrument (MettlerToledo Inc). The measurements were performed up to $80^{\circ} \mathrm{C}$ with a scanning rate of $5{ }^{\circ} \mathrm{C} / \mathrm{min}$.

Fluorescence Images of mPEG-PCDA Liposome Solution. The mPEG-PCDA/PCDA liposome solutions were heated at different temperatures, and fluorescence images were obtained using a fluorescence microscope (Nikon Eclipse TS100).

\section{Results and Discussion}

Polymerization by UV Irradiation. A schematic representation of the formation of $10 \mathrm{~mol} \% \mathrm{mPEG}-\mathrm{PCDA}$ liposomes in aqueous solution is shown in Figure 1. The general method for preparing PCDA liposomes typically requires heating at $80^{\circ} \mathrm{C}$ prior to sonication, as PCDA has a melting point of $65^{\circ} \mathrm{C}$ and is poorly soluble in water. In contrast, as the system described here includes PEG, which has good water solubility, mPEG-PCDA liposomes could be prepared without the need for heating. As the liposomes were formed from the polymeric lipid monomers under UV irradiation $(254 \mathrm{~nm})$, the solution was observed to change from colorless to blue color (Figure 3(b)).

Characterization of 10 mol \% mPEG-PCDA Liposomes using DLS. As shown in Table 1, the mean size of the

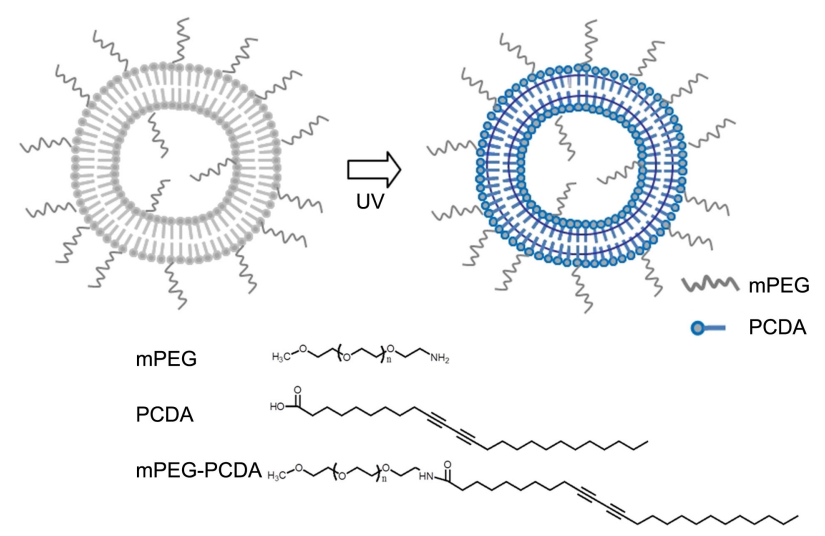

Figure 1. Schematic representation of the polymerization of 10 mol \% mPEG-PCDA liposome.

Table 1. Dynamic light scattering and zeta potential data of 10 mol \% mPEG-PCDA and PCDA liposomes

\begin{tabular}{cccc}
\hline & \multicolumn{2}{c}{ Mean diameter $(\mathrm{nm})$} & $\begin{array}{c}\text { Zeta potential } \\
(\mathrm{mV})\end{array}$ \\
\cline { 2 - 3 } & before UV & after UV & \\
\hline $\begin{array}{c}\text { PCDA liposome } \\
10 \text { mol\% mPEG-PCDA } \\
\text { liposome }\end{array}$ & $104.3 \pm 0.42$ & $80.3 \pm 0.16$ & $-16.4 \pm 2.00$ \\
\hline
\end{tabular}

prepared mPEG-PCDA liposomes was found to be similar to that of native PCDA liposomes, with no significant alteration occurring upon UV irradiation. This observation is assumed to be due to an improvement in solubility by introducing PEG, as the poorly water-soluble PCDA permits the liposomes to easily aggregate.

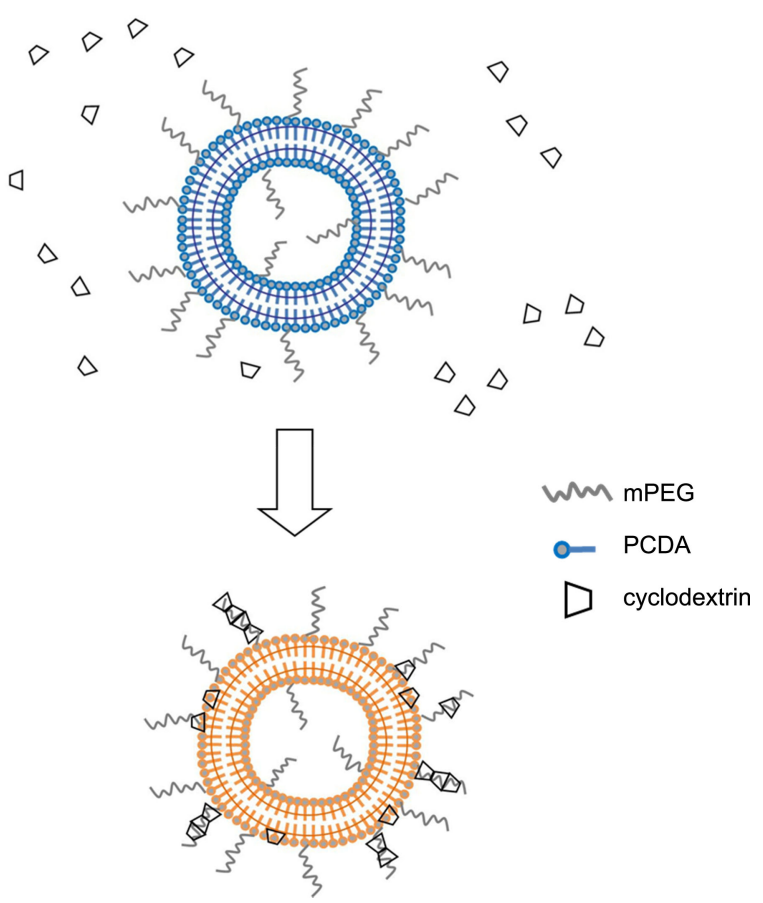

Figure 2. Schematic illustration of $10 \mathrm{~mol} \% \mathrm{mPEG}-\mathrm{PCDA}$ liposome upon addition of $\alpha$-cyclodextrin. 


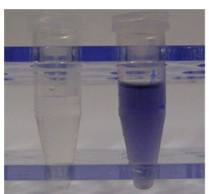

(a) (b)

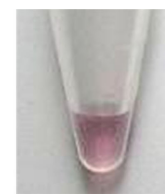

(c) (e)

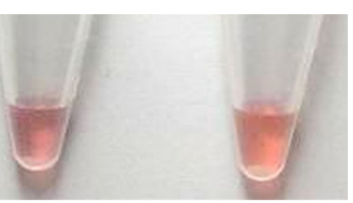

Figure 3. Photographs of $10 \mathrm{~mol} \% \mathrm{mPEG}$-PCDA liposomes before (a), and after (b) UV irradiation. Color transition of polymerized $10 \mathrm{~mol} \% \mathrm{mPEG}$-PCDA liposomes caused by the addition of $\alpha$-cyclodextrin at 20:1 (c), 41:1 (d), and 82:1 (e) molar ratios ( $\alpha$-cyclodextrin/PCDA), respectively.

In addition to size, surface charges of the liposomes were measured and are displayed in Table 1. The $10 \mathrm{~mol} \%$ mPEG-PCDA liposome and the native PCDA liposome were observed to have similar zeta potential values.

Interaction of PDA and $\boldsymbol{\alpha}$-Cyclodextrin. Figure 2 shows a schematic representation of polymerized $10 \mathrm{~mol} \% \mathrm{mPEG}-$ PCDA liposomes upon addition of $\alpha$-cyclodextrin, illustrating the head-to-head and tail-to-tail arrangement previously proposed by the Kamachi group. ${ }^{25}$ The process of inclusion complex formation between mPEG and $\alpha$-cyclodextrin involves the formation of hydrogen bonds between the oligosaccharide units with the neighboring molecule. Here, the polymerized liposome solution became turbid immediately upon addition of $\alpha$-cyclodextrin, followed by a color change from blue to red. In addition, the degree of turbidity of the solution decreased as the amount of added $\alpha$-cyclodextrin increased (Figure 3). This is consistent with previous reports that linear low-molecular weight PEG forms crystalline inclusion complexes with $\alpha$-cyclodextrin.

DSC Measurements. DSC curves for the mPEG-PCDA and control samples are shown in Figure 4. Melting points for the native PCDA and mPEG-PCDA appeared at 64.04 ${ }^{\circ} \mathrm{C}$ and $55.83{ }^{\circ} \mathrm{C}$, respectively, while no distinct peaks were present in the curves for the pure $\alpha$-cyclodextrin or the solid precipitate. Based on the results, introduction of PEG appeared to be responsible for this decrease in melting point.

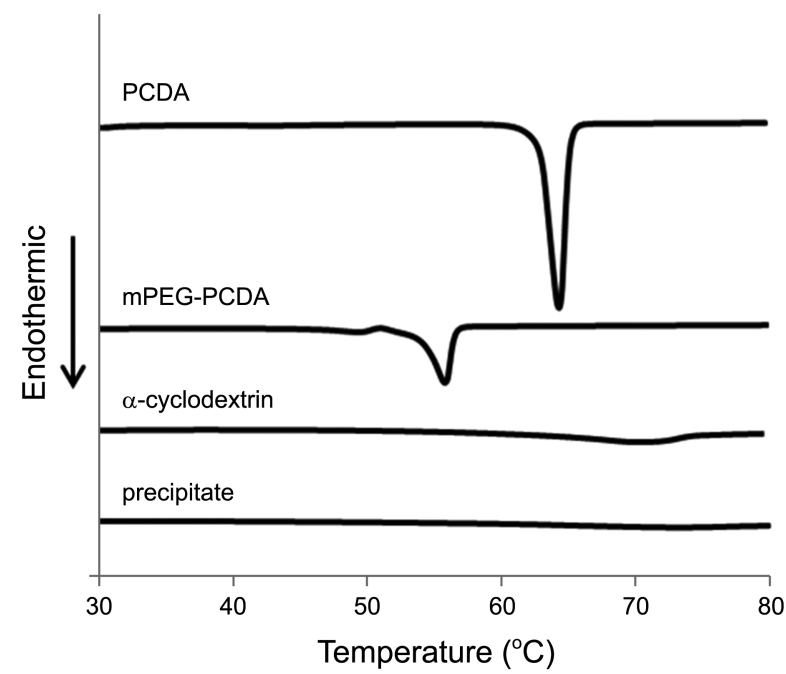

Figure 4. DSC thermograms of native PCDA, mPEG-PCDA, $\alpha-$ cyclodextrin, and the formed precipitates.
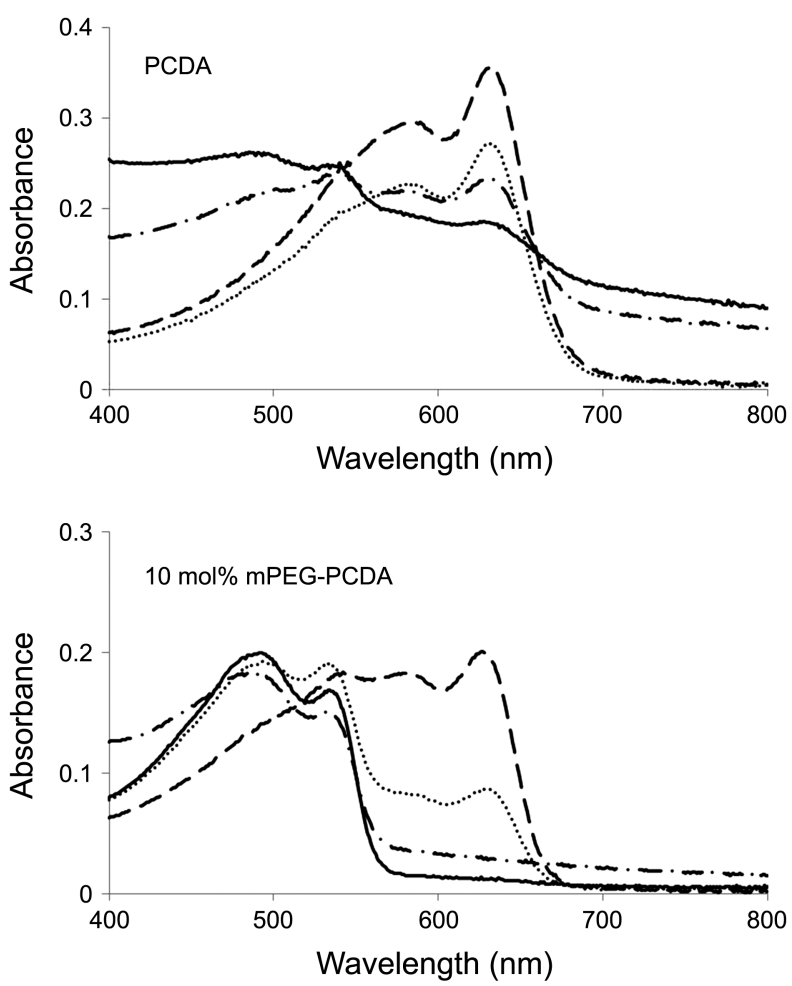

Figure 5. Wavelength scan results on the addition of different amounts of $\alpha$-cyclodextrin. Molar ratio ( $\alpha$-cyclodextrin/PCDA) 0:1 (dashed line), 20:1 (dotted line), 41:1(dashed-dotted line), 82:1 (solid line).

The scans also confirmed that no free PCDA or mPEGPCDA lipids were present in the white precipitate.

UV-vis Spectroscopy. The UV-vis spectra of the liposomes in aqueous solution are shown in Figure 5. In the absence of cyclodextrin, the maximum absorption band could be observed at $640 \mathrm{~nm}$ after irradiation with $254 \mathrm{~nm}$ UV light for both the mPEG-PCDA and PCDA control sample, suggesting that the PEG-conjugated PCDA liposome backbones have similar chromic properties to the native PCDA.

UV spectra for samples formed upon incubation of liposomes with $\alpha$-cyclodextrin at molar ratios of 20:1, 41:1, and $82: 1$ (final concentrations of $0.09,0.29$, and $0.5 \mathrm{mM} \alpha-$ cyclodextrin, respectively) exhibited perturbations because of inclusion complex formation between the PEG chain of the polymerized vesicle and the $\alpha$-cyclodextrin.

In the case of native PCDA liposomes, the absorption maximum peak shifted from $640 \mathrm{~nm}$ (blue phase) to $540 \mathrm{~nm}$ (red phase) at a 41:1 molar ratio ( $\alpha$-cyclodextrin/PCDA), with the peak at $640 \mathrm{~nm}$ still remaining evident even at 82:1. However, for the $10 \mathrm{~mol} \% \mathrm{mPEG}-\mathrm{PCDA}$ liposomes, a blue shift owing to inclusion complex formation could be seen, even at a molar ratio of 20:1. Moreover, above a ratio of $41: 1$, no peak was present at $640 \mathrm{~nm}$ due to completion of color change; it could be thus confirmed that PEG introduction improves liposome sensitivity and stability, which can be attributed to an increase in solubility and improved complex formation between PEG and $\alpha$-cyclodextrin superior to that of carboxyl group-containing PCDA. 


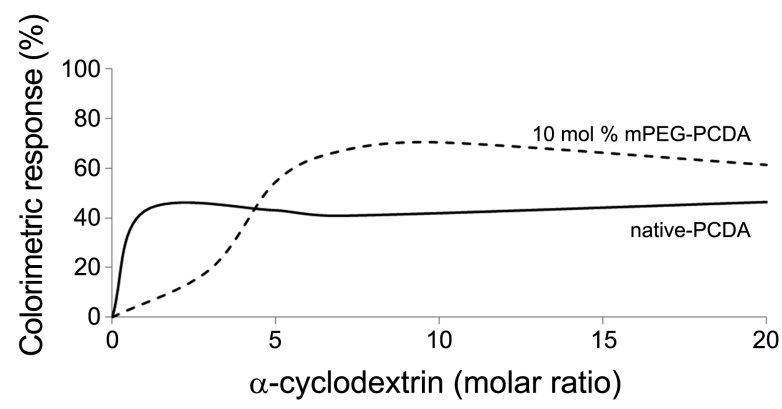

Figure 6. CR (\%) of native PCDA and $10 \mathrm{~mol} \% \mathrm{mPEG}-\mathrm{PCDA}$ following addition of $\alpha$-cyclodextrin.

Colorimetric Response. To evaluate the color changes upon the addition of $\alpha$-cyclodextrin to mPEG-PCDA, the well-known equation of colorimetric response (CR) was utilized as follows:

$$
\mathrm{CR}(\%)=\left(\mathrm{PB}_{0}-\mathrm{PB}_{\mathrm{f}}\right) / \mathrm{PB}_{0} \times 100
$$

where $\mathrm{PB}_{0}$ and $\mathrm{PB}_{\mathrm{f}}$ are the percent of blue before and after the color transition, respectively, with $\mathrm{PB}$ calculated as follows:

$$
\mathrm{PB}=\mathrm{A}_{640 \mathrm{~nm}} /\left[\mathrm{A}_{640 \mathrm{~nm}}+\mathrm{A}_{540 \mathrm{~nm}}\right] .
$$

Therefore, when the blue-to-red color transition is complete, the theoretical CR value is $100 \%$. As shown in Figure 6 , addition of $\alpha$-cyclodextrin to the mPEG-PCDA liposome solution resulted in $\mathrm{CR}$ saturation at approximately $70 \%$ at a 1:6 molar ratio (PCDA: $\alpha$-cyclodextrin). However, in comparison to the native PCDA, the color transition was fairly slow at low $\alpha$-cyclodextrin concentrations. It is possible that in the absence of PEG, the stimulation caused by docking of the $\alpha$-cyclodextrin structure to the liposome might be transferred quickly to the backbone, causing a rapid color change. The maximum CR value achieved for PCDA alone was only around $40 \%$, demonstrating that the blue-to-red color transition was not significant. Based on these observations, introduction of PEG appeared to be responsible for the observed increase in color transition.

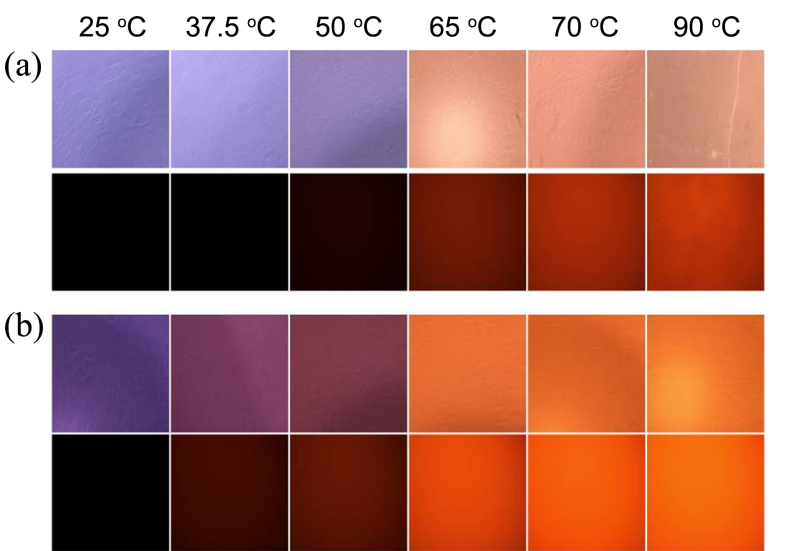

Figure 7. Temperature-dependent optical (top panel) and fluorescent (bottom panel) images of native PCDA (a) and $10 \mathrm{~mol} \%$ mPEG-PCDA liposomes (b).
Thermochromism and Fluorogenic Properties of 10 mol \% mPEG-PCDA Liposomes. It is known that PCDA liposomes exhibit unique fluorogenic properties in response to heat; accordingly, a comparison of fluorescence intensity was made between the $10 \mathrm{~mol} \% \mathrm{mPEG}-\mathrm{PCDA}$ and the native PCDA liposomes (Figure 7). The blue-to-red color transition was observed for the native PCDA liposomes above $65{ }^{\circ} \mathrm{C}\left(c f . T_{\mathrm{m}}=63{ }^{\circ} \mathrm{C}\right)$. However, the $10 \mathrm{~mol} \%$ PEGPCDA liposomes presented a blue-to-red color change and red fluorescence which started at $37.5^{\circ} \mathrm{C}$ and was apparent at $50{ }^{\circ} \mathrm{C}$ and above, providing further evidence that the optical and fluorescence sensitivity was increased in a temperature-dependent manner. The introduction of PEG chains appears to have the effect of increasing thermosensitivity of the native PCDA liposomes. These properties provide the PEG-PCDA liposome with great potential for use in applications such as sensitive PDA-based biosensors or chemosensors.

Acknowledgments. This research was supported by Basic Science Research Program through the National Research Foundation of Korea (NRF) funded by the Ministry of Education, Science and Technology (20100021647).

\section{References}

1. Vyas, A.; Saraf, S. Journal of Inclusion Phenomena and Macrocyclic Chemistry 2008, 62, 23.

2. Tiwari, G.; Tiwari, R.; Rai, A. K. J Pharm. Bioallied Sci. 2010, 2 , 72.

3. Ogoshi, T.; Harada, A. Sensors 2008, 8, 4961.

4. Sabadini, E.; Cosgrove, T. Langmuir 2003, 19, 9680.

5. Szejtli, J. Chemical Reviews 1998, 98, 1743.

6. Harada, A.; Kamachi, M. Macromolecules 1990, 23, 2821.

7. Jiao, H.; Goh, S. H.; Valiyaveettil, S. Macromolecules 2002, 35, 1980.

8. Choi, H.; Bae, Y. M.; Yu, G. S.; Huh, K. M.; Choi, J. S. Journal of Nanoscience and Nanotechnology 2008, 8, 5104.

9. Okada, S.; Peng, S.; Spevak, W.; Charych, D. Accounts of Chemical Research 1998, 31, 229.

10. Charych, D. H.; Nagy, J. O.; Spevak, W.; Bednarski, M. D. Science 1993, 261, 585.

11. Yuan, Z. Z.; Lee, C. W.; Lee, S. H. Angewandte Chemie-International Edition 2004, 43, 4197.

12. Ahn, D. J.; Chae, E. H.; Lee, G. S.; Shim, H. Y.; Chang, T. E.; Ahn, K. D.; Kim, J. M. Journal of the American Chemical Society 2003, 125, 8976.

13. Beckham, H. W.; Rubner, M. F. Macromolecules 1993, 26, 5198.

14. Yoon, J.; Chae, S. K.; Kim, J. M. Journal of the American Chemical Society 2007, 129, 3038.

15. Bloor, D. Macromol. Chem. Phys. 2001, 202, 1410.

16. Carpick, R. W.; Sasaki, D. Y.; Marcus, M. S.; Eriksson, M. A.; Burns, A. R. Journal of Physics-Condensed Matter 2004, 16, R679.

17. Nallicheri, R. A.; Rubner, M. F. Macromolecules 1991, 24, 517.

18. Tomioka, Y.; Tanaka, N.; Imazeki, S. Journal of Chemical Physics 1989, 91, 5694.

19. Ahn, D. J.; Kim, J. M. Accounts of Chemical Research 2008, 41, 805.

20. Kim, J. M.; Lee, J. S.; Woo, S. Y.; Ahn, D. J. Macromol. Chem. Phys. 2005, 206, 2299.

21. Gill, I.; Ballesteros, A. Angewandte Chemie-International Edition 2003, 42, 3264.

22. Kolusheva, S.; Kafri, R.; Katz, M.; Jelinek, R. Journal of the 
American Chemical Society 2001, 123, 417.

23. Okada, S. Y.; Jelinek, R.; Charych, D. Angewandte ChemieInternational Edition 1999, 38, 655.
24. Ma, Z. F.; Li, J. R.; Liu, M. H.; Cao, J.; Zou, Z. Y.; Tu, J.; Jiang, L. Journal of the American Chemical Society 1998, 120, 12678.

25. Harada, A.; Li, J.; Kamachi, M. Macromolecules 1993, 26, 5698. 